\title{
Seasonal cycle of cloud cover analyzed using Meteosat images
}

\author{
J. Massons ${ }^{1}$, D. Domingo ${ }^{1}$, J. Lorente ${ }^{2}$ \\ ${ }^{1}$ Lab. Física Aplicada, Univ. Rovira i Virgili. Pl. Imperial Tarraco 1, E-43005 Tarragona, Spain \\ ${ }^{2}$ Dept. Astronomia i Meteorologia. Univ. Barcelona. Avda. Diagonal 647, E-08028 Barcelona, Spain
}

Received: 8 October 1996 / Revised: 8 September 1997 / Accepted: 9 September 1997

\begin{abstract}
A cloud-detection method was used to retrieve cloudy pixels from Meteosat images. High spatial resolution (one pixel), monthly averaged cloud-cover distribution was obtained for a 1-year period. The seasonal cycle of cloud amount was analyzed. Cloud parameters obtained include the total cloud amount and the percentage of occurrence of clouds at three altitudes. Hourly variations of cloud cover are also analyzed. Cloud properties determined are coherent with those obtained in previous studies.
\end{abstract}

Key words. Cloud cover $\cdot$ Meteosat

\section{Introduction}

Clouds have a powerful influence on the radiation budget because they provide the greatest contribution to the scattering of solar radiation, enhancing the albedo of the earth-atmosphere system. Randall et al. (1984) estimate that a $4 \%$ increase in global low-cloud fraction could offset a $2-3 \mathrm{~K}$ rise in global temperature due to a doubling of $\mathrm{CO}_{2}$. However, the full role of clouds in controlling the current climate is still far from understood, and remains one of the main causes of uncertainty in climate modelling. In order better to use satellite images for analyzing cloud distributions, many works have been devoted to cloud detection by separating the satellite observations into clear and cloudy categories (Saunders, 1986; Rossow and Garder, 1993).

Although the measurement of cloudiness poses many problems, the need to study clouds and predict them correctly is widely recognized. As pointed out by several authors (Slingo, 1987) the major problem in the validation of cloudiness is the difference in definition between models and observations. Surface observations have

Correspondence to: Dr. J. Massons

e-mail: massons@quimica.urv.es been compiled from many years of observation (Warren et al., 1986, 1988). In recent years these data have been supplemented by satellite observations, which provide better global distribution and spatial resolution. In addition to providing poor spatial coverage, surface observations cannot view the cloud top, which is the part of the cloud reflecting solar radiation and emitting thermal radiation. Satellite radiometers view the cloud tops and are able to produce a global data set of spatially averaged cloud parameters.

Following Rossow et al. (1985), there are several limitations on the evaluation of the cloud amount using satellite data. Among them must be cited the limitations in global uniformity and space and time resolution of the data and the lack of a "truth" data set against which to compare the results of each algorithm in order to judge the performance of each method. The advantages of a cloud climatology derived from satellite data have led to the implementation of important international programs such as the International Satellite Cloud Climatology Project (ISCCP) (Rossow and Schiffer, 1991; Drake, 1993). ISCCP has created a global climatology by combining data collected by both geostationary and polar orbiting satellites. The data used in this research span the years 1984-1990. The cloud-detection process is discussed in detail in Rossow and Garder (1993). The pixel level results are averaged to an equalarea grid with spatial resolution of about $280 \mathrm{~km}$ to produce cloud fraction and average cloud properties at 3-h intervals. These data have been used to analyze the diurnal variation of cloudiness over large areas (Rossow and Schiffer, 1991; Kondragunta and Gruber, 1994; Rozendal et al., 1995). Ground-based observations of clouds include classification by morphology and base height above the local terrain (Warren et al., 1986, 1988). Classification made from satellite observations uses the division of clouds by their top height and/or optical thicknesses (Minnis and Harrison, 1984; Desbois and Sèze, 1984; Pankiewicz, 1995; Massons et al., 1996). In the present paper the temporal and spatial variations of cloud fractions are investigated using satellite data 
provided by Meteosat for one complete year. The analysis is performed at 12:00 GMT with a resolution of one pixel (about $7 \times 5 \mathrm{~km}$ ). In recent years, great attention has been paid to the analysis of the cycles of cloud fraction, both seasonal and diurnal (Minnis and Harrison, 1984; Duvel and Kaudel, 1985; Warren et al., 1985; Rossow and Lacis, 1990; Rossow and Schiffer, 1991; Thiao and Turpeinen, 1992; Drake, 1993; Carlston and Wolf, 1993; Klein and Hartmann, 1993; Norris and Leovy, 1994). Most of them use ISCCP data averaged over large spatial regions or are restricted to only a short period. Data available through ISCCP provide a high number of global cloud data whose full potential will take time to be discovered. Unfortunately, this does not allow a pixel-scale spatial analysis; this requirement is essential for the study of small-scale cloud structures and/or heterogeneous land areas, and demands a complementary higher-resolution data base. There have been many assessments of the effect of image pixel size on cloud cover determination (see references in Rossow et al., 1985, 1993). So, for example, the analysis of subpixel-size clouds such as cumulus indicates (Rozendaal et al., 1995) that missing small cumulus clouds because of their spatial scale and small effects on thermal infrared radiances will produce substantial errors in the statistical analysis of the cloud field.

The analysis of the satellite data is described in Sect. 2. In particular it includes a brief description of the cloud-detection scheme used. As Rossow et al. (1985) pointed out, the largest differences between clouddetection methods occur in areas where the surface properties varied rapidly in time or over small spatial scales. Areas where a significant portion of clouds were either very low level and broken or optically very thin also require a specific treatment. In order to increase the accuracy, all the algorithms should be made sensitive to the presence of clouds in low contrast situations. So, in the present study an algorithm based on the analysis of spatial and time variations in the image was used. The method follows the work of Coakley and Bretherton (1982) which uses the local spatial variance to separate the homogeneous regions from intermediate points, interpreted as a partially covered field of view. The Coakley and Bretherton method uses the general observation that clear regions exhibit smaller spatial variations than do broken cloud regions. This methodology was also applied by Coakley and Baldwin (1984) for analyzing fair-weather and trade-wind cumulus. The main results obtained from the application of the algorithm are presented in Sect. 3. They include comparisons of the cloud amount obtained here and ISCCP data, as well as a series of plots describing the seasonal variation of the parameters analyzed.

\section{Satellite data and preprocessing}

The images used in the present work correspond to Meteosat digital images. Images correspond to a window region of $512 \times 512$ pixels centered on the Iberian Peninsula and cover the domain shown in Fig. 1.
Visible (VIS) and thermal infrared (IR) images of 12:00 GMT from August 1994 to July 1995 were analyzed. The effects on the radiance of satellite viewing angle and solar illumination geometries were reduced using a standard Lambertian correction model, i.e., dividing all VIS counts in the image by the cosine of the solar zenith angle. Hereafter, VIS data will refer to the VIS corrected image. The conversion from IR counts to radiance is made using the coefficients stored in the calibration block of the header. Radiance is then converted to brightness temperature using the inverse of the Planck function. Working with temperatures, instead of IR count numbers, has the advantage of avoiding changes due to the variations of the thermal calibration parameters. This allows images from different days to be accurately compared. Hereafter IR data will refer to brightness temperature computed from IR grey level.

Two kinds of result will be presented here: monthly averaged time-evolution of percentage of occurrence of clouds over selected areas of the image, and seasonally averaged cloud amount over the whole analyzed window. For the first, cloud amount was analyzed in some detail at points of the line $\mathrm{AB}$ (see Fig. 1). This line includes sea areas and land points and is especially interesting for analysis of the spatial and temporal variability of the cloudiness. The analysis is particularized for the points $a, b$, and c. These points, located over the AB line, correspond both to sea areas (one located over the Mediterranean and the another over the Atlantic) and land surfaces (over Spain). Seasonally averaged cloud data will not be presented in the shaded area located in the northern part of the analyzed window (Fig. 1). Data at those high latitudes have significant errors due to the low sun elevation angles during a great part of the year.

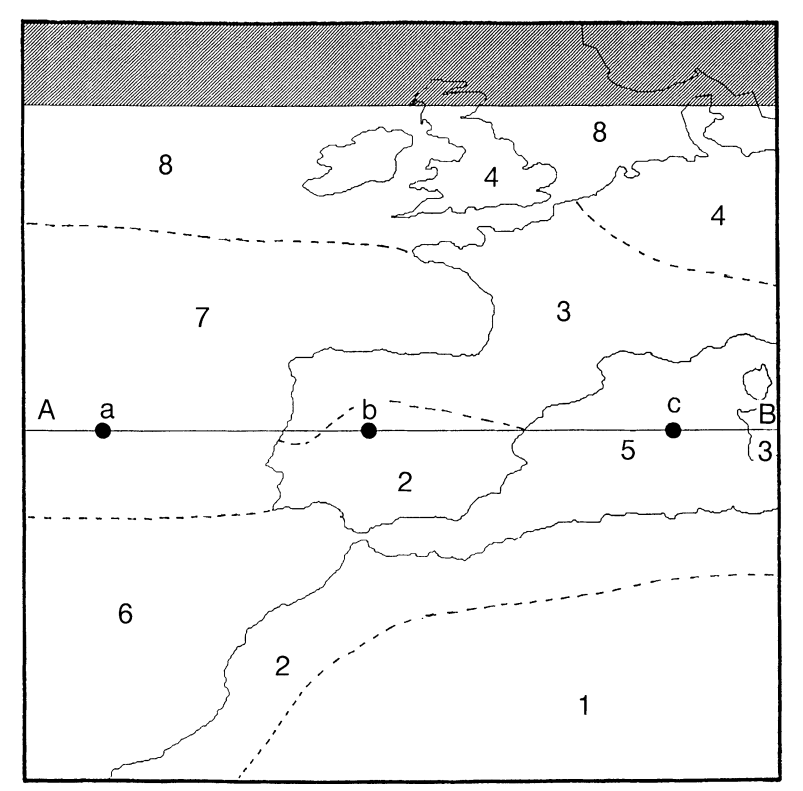

Fig. 1. Schematic view of the analyzed area; symbols are explained in the text 
In the first step of the image processing, clouds were isolated using a cloud-detection method. This method uses bispectral VIS-IR histograms (Sèze and Desbois, 1987; Sèze and Rossow, 1991) together with spatial variance (Coakley and Bretherton, 1982) and short-term time-series to detect cloudy pixels in the image. As established by Rossow et al. (1985), the diversity of conditions encountered on earth precludes the use of any one method everywhere. A successful global cloud algorithm must be situation independent and employ a series of tests to retain flexibility. For this reason, the cloud-detection algorithm used here employs both space and time contrast tests as well as radiance threshold methods (both in IR and VIS images). The method uses the VIS-IR image pair corresponding to the instant to be analyzed and the image pair of the previous hour. It must be indicated that the time contrast test has been found more effective than the spatial variant. Summarizing, the method divides the image into eight geographical areas (1-8, see Fig. 1) more or less homogeneous, and uses a temporal coherence function between the VIS-IR images to be analyzed and those of $1 \mathrm{~h}$ earlier. The first step in the processing involves the computation of the time composite coherence function between the VIS (and IR) image to be analyzed and the one corresponding to $1 \mathrm{~h}$ earlier. The temporal coherence function at pixel $(i, j)$ for VIS images is defined as: the most difficult to be detected. As already indicated, not all clear pixels are found to have high coherence values, but using the present method only a representative portion of clear pixel population is needed to run the detection procedure. The following step in the processing involves the computation of $\mathrm{H} 2 \mathrm{D}$ for each one of the eight geographically homogeneous zones of the image. Only the points having a high VIS and IR coherence are used. After that, the warmest and darkest class in the H2D is located. Each pixel of the image having a brightness temperature (or VIS grey level) greater than (or lower than) the elements of that class is considered clear. This step collects the clear pixels that have not been detected in the first step (temporal coherence method). Several authors have noted the difficulties of the cloud-detection process in coastlines (Desbois and Sèze, 1984; Saunders, 1986). For this reason, every point belonging to a three-pixels-wide coastline is analyzed again in more detail. If it is classified as cloudy, the algorithm computes the most populated class in the $5 \times 5$ neighboring pixels (outside the coastline) and the current pixel is assigned to this class. On the other hand, if the pixel is initially classified as clear, then the label of the pixel is not modified and no other calculations are realized with it. Finally, it must be indicated that pixels classified as clear in the 1-hearlier image and cloudy in the current image are tested

$$
C O(i, j)=\frac{\sum_{k, j, m=-1}^{1}\left(\operatorname{VIS}_{1}(i+k, j+m)-\overline{\operatorname{VIS}_{1}(i, j)}\right) \overline{\left(\operatorname{VIS}_{2}(i+k, j+m)-\overline{\operatorname{VIS}_{2}(i, j)}\right)}}{\sqrt{\sum_{k, j, m=-1}^{1}\left(\operatorname{VIS}_{1}(i+k, j+m)-\overline{\operatorname{VIS}_{1}(i, j)}\right)^{2} \sum_{k, j, m=-1}^{1}\left(\operatorname{VIS}_{2}(i+k, j+m)-\overline{\operatorname{VIS}_{2}(i, j)}\right)^{2}}},
$$

where the subscript 1 indicates values evaluated in the image of the analyzed hour and subscript 2 refers to data obtained from the images of $1 \mathrm{~h}$ earlier. Overbars indicate spatial mean values computed averaging the grey levels on a $3 \times 3$ window centered on the computed pixel. A similar calculation is realized for IR images. Both VIS and IR temporal coherence functions measure the temporal variability of the pixel. If a pixel is clear in both the present-time and 1-h-earlier images, these two coefficients show high values. If the pixel is cloudy in the present-time and/or in the previous image, a greater variability is introduced, which produces lower values of the coherence functions. The pixels having a low temporal variability in both VIS and IR channels are used as applicants for clear pixels and are used to build the bidimensional VIS-IR histogram (H2D) for highcoherence pixels. Pixels having low temporal variability are not necessarily clear because static cloud clusters are also detected by this operator. A new step in the process is needed in order to eliminate cloudy pixels from the H2D obtained (and add clear pixels not detected by the first calculation). However, it must be indicated that the time coherence filter runs acceptably well, mainly because it allows the elimination of almost all partially covered pixels and broken clouds, which are for being assigned to mixed or undecided pixels (they usually correspond to broken clouds, edges of great cloud formations, etc.). For these pixels, the variance of the VIS and IR data is computed using a $3 \times 3$ window centered on the pixel. If the variance in the VIS and or IR channel is greater than 20 units, the pixel is classified as mixed. Otherwise it remains in the cloudy class. The production of a cloud mask for a typical pair of images requires less than $40 \mathrm{~s}$ on a Pentium PC running at $160 \mathrm{MHz}$. The more consuming time process is the computation of the time coherence (about $60 \%$ ). Cloud detection performs well, not only at noon, but at other hours of the day.

As an example, Fig. 2a shows the time-evolution of 12:00 GMT VIS and IR data from August to December 1994 for point b, located over Spain. Points labelled ' 0 ' correspond to pixels detected as clear, ' 2 ' indicates cloudy pixels and ' 1 ' represents mixed or undecided pixels; these pixels were not clearly identified as cloudy or cloud-free using the thresholds. As can be observed, the proportion of the last group is relatively small (about $7 \%$ ). In this work this last group of pixels were treated as cloudy. Note the presence of cloudy pixels with VIS or IR data values closer to the ones corresponding to clear pixels. This similarity in grey 


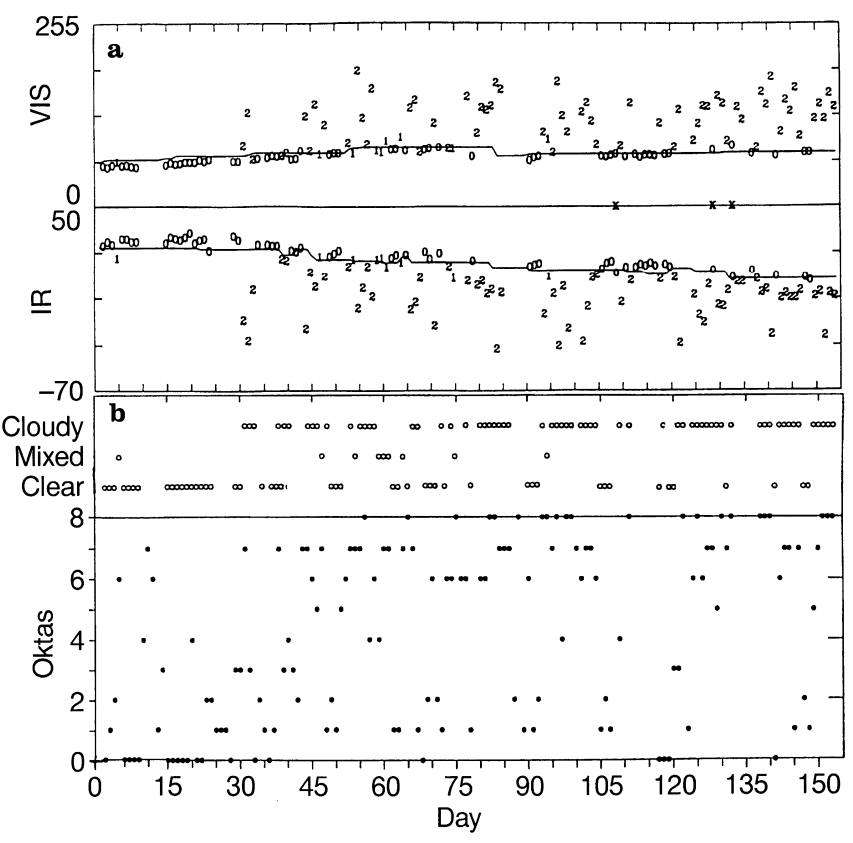

Fig. 2. a Time-evolution of VIS and IR data for August-December 1994 and for point b. The legend is explained in the text. b Surface observations in oktas of the cloudiness for point $b$

levels, which occurs in semitransparent high-level clouds or low-level stratus, hinders the use of cloud-detection algorithms. Days with no data in Fig. 2 were days for which the image of 11:00 or 12:00 GMT were not in our hourly image data base.

As an indicator of cloud-detection performance, these short-term time-series of VIS and IR values were numerically treated in order to determine the thresholds to be used as separators between cloudy and clear pixels. Analyzing the frequency histograms of the data in a medium-term series of 31 days (15 before and 15 after the analyzed day), a standard class separation method was used to detect the threshold separating cloud and clear pixels. The VIS and IR threshold levels determined in this way are plotted in Fig. 2a as solid lines. So, the relative value of the VIS-IR data indicates whether the pixel is detected clear or cloudy by this time-evolution method. Few disagreements between both assignments (method used and medium-term time-series analysis) were found, showing the power of the method. These disagreements are labelled as ' $x$ '. The amount of disagreement is relatively small (about $2 \%$ of the total). This amount is even smaller over sea surfaces, where the grey level of the clear pixels is more homogeneous. The cloud-detection method requires few computer resources and little a priori information (only the 1-h-previous VIS-IR image pair).

A comparison of the satellite-retrieved cloudiness for August-December 1994 with surface observations for point b (see Fig. 1) are presented in Fig. 2b. Surface observation data were supplied in okta code by the Instituto Nacional de Meteorología, the national meteorological service of Spain, and correspond to the synoptic surface station of Zamora, Spain (station number 81300 , located at $41.30^{\circ} \mathrm{N}, 5.44^{\circ} \mathrm{W}$ ). All cloud data were measured at 12:00 GMT. The upper part of the figure also includes the class, determined by the cloud-detection algorithm. A general agreement between surface observations and satellite derived cloudiness can be observed, but, as indicated by Sèze et al. (1983), it is almost impossible to make general statements about the level of agreement between surface and satellite observations of cloud amounts, especially as the area over which the comparation was undertaken is relatively small and suffers from rapidly changing weather. Also, the different point of view of both observations and the different area cover make difficult the direct comparison between these data. As indicated by Henderson-Sellers et al. (1987), the area of vision of a surface observer is $100 \times 100 \mathrm{~km}$. This fact produces significant differences between surface and (one pixel) satellite observations when the cloud field is nonuniform. Table 1 summarizes the preceding comparison. The number of occurrences of every octa value is computed for each pixel label (clear/mixed/cloudy) both for low and total cloudiness. The results indicate that almost all of the cases that are determined as clear by the cloud-detection algorithm correspond to low okta measurements and that the higher octa values are associated with cases seen as cloudy by the algorithm. Taking into account the above mentioned reasons, the agreement between surface and satellite observations is considered reasonable.

\section{Results obtained}

In order to test the accuracy of the cloud-detection program, the results provided by the program for total cloud amount at 12:00 GMT were compared with ISCCP C1 data obtained for the 7-year period 19841990. For each pixel and each month, the total cloud amount is computed as the fraction of days during which the pixel is seen as cloudy by the cloud-detection program. Cloud amount is expressed as a percentage and corresponds to a time-averaged cloud-cover fraction over the pixel. The comparison between yearly averaged ISCCP data and the present data spatially averaged onto the ISCCP grid shows a general agreement between both data sets. So, for example, the averaged RMS of the differences between total cloud amount in the grid points for March, July, and November are respectively 8, 7, and 12 (over 100). These differences can be considered sufficiently low, taking into account that

Table 1. Number of occurrences of okta values (total and low-level clouds) for each clear-mixed-cloudy situation for point $b$

\begin{tabular}{lrrrrrrrrr}
\hline Octas & 0 & 1 & 2 & 3 & 4 & 5 & 6 & 7 & 8 \\
\hline total & & & & & & & & & \\
Clear pixels & 17 & 12 & 10 & 3 & 3 & 0 & 1 & 2 & 0 \\
Mixed pixels & 0 & 1 & 0 & 0 & 1 & 1 & 3 & 2 & 1 \\
Cloudy pixels & 1 & 2 & 0 & 4 & 3 & 2 & 12 & 21 & 20 \\
low & & & & & & & & & \\
Clear pixels & 4 & 3 & 3 & 1 & 1 & 0 & 1 & 0 & 0 \\
Mixed pixels & 1 & 2 & 1 & 0 & 2 & 1 & 0 & 0 & 0 \\
Cloudy pixels & 2 & 2 & 0 & 3 & 5 & 13 & 10 & 12 & 9 \\
\hline
\end{tabular}



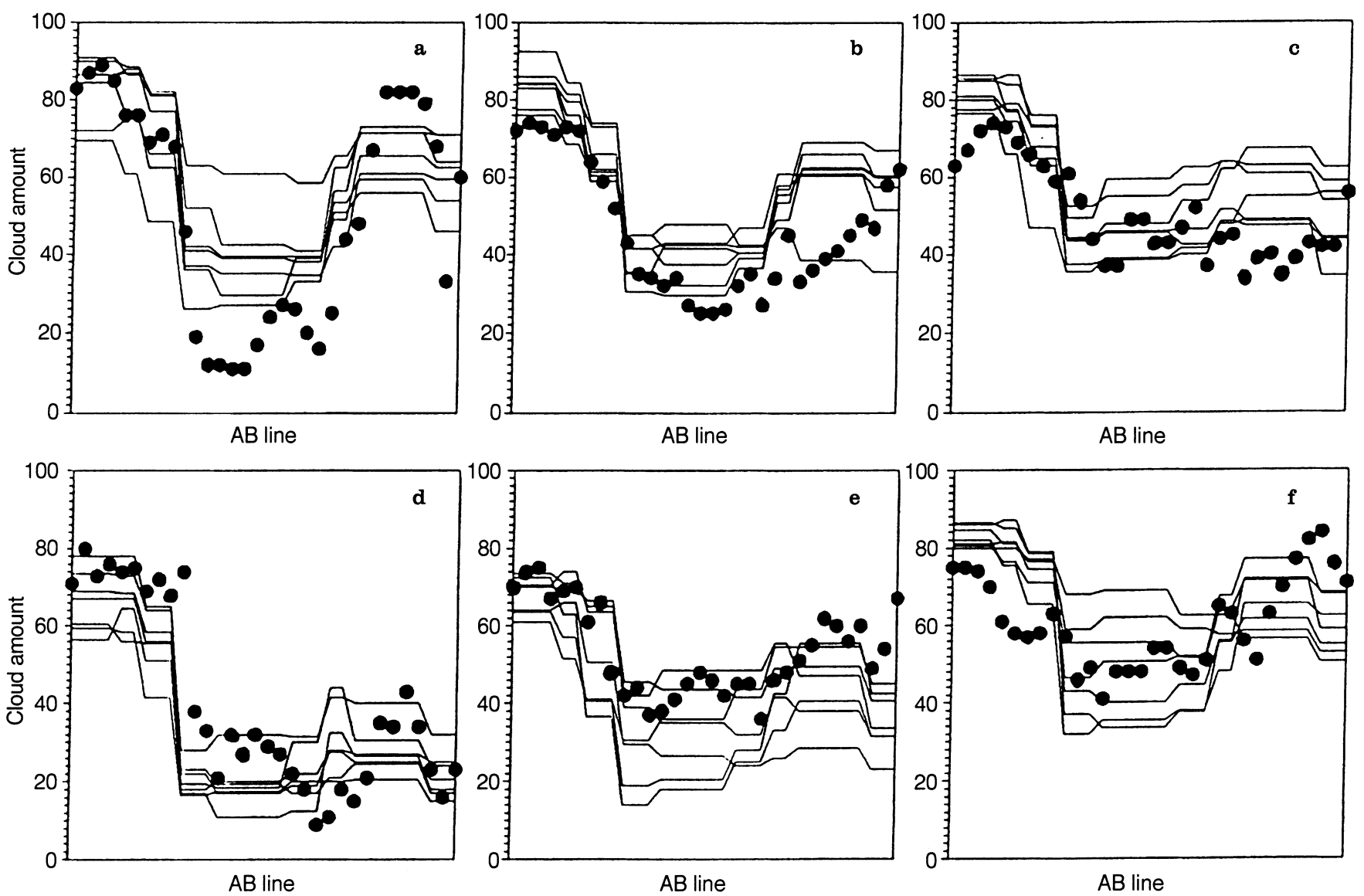

Fig. 3a-f. Total cloud amount for the line section AB for different months. a January, b March, c May, d July, e September, f November. Solid lines: ISCCP data. Symbols: data computed in the present work

the data correspond to different years and the great temporal and spatial variability of the cloud field.

These two data fields are compared in more detail over the $\mathrm{AB}$ line (see Fig. 1). The cloud amounts determined for the $\mathrm{AB}$ line are depicted in Fig. 3 for January, March, May, July, September, and November. ISCCP data are represented as solid lines (one line for every year) and symbols are used to plot the cloudamount data obtained here. In order to increase the legibility of the plot, only one point is presented for every 16 data values obtained (32 points for 512 data values). The plots show that the obtained cloud amount reproduces the behavior of the ISCCP data acceptably. This fact indicates that the cloud-amount data obtained for the time-period analyzed are representative of the climatology of the area and that the cloud-detection algorithm correctly captures the threshold level separating cloudy and cloud-free pixels. Cloud amount over the ocean has a maintained behavior with cloud amount of around $70-80 \%$, more or less independent of the season. Cloud amount over Spain is less (with cloud-amount data ranging from $10-20 \%$ to $40-50 \%$ ), with minimum values in winter and summer. Finally, cloud amount over the Mediterranean Sea is generally higher than over land, having pronounced minimum values during summer months. Minor differences between ISCCP data and the present data can be easily explained by the spatial and temporal variability of the cloud-amount data distribution, especially over land.

The monthly time-evolution of the cloud-cover data is presented in Fig. 4 for the three points a, b, and c (Fig. 1). ISCCP data for these points are also included. Again, the seasonal behavior of the obtained data agrees reasonably with ISCCP results. The data tendency agrees with the preceding comments: high and maintained cloud-amount values over points located on the ocean, and more season-dependent values over the Mediterranean Sea and (especially) over the land area. Strong gradients of percentage of occurrence of clouds can be observed in the coastal areas, especially on the Atlantic coastlines. Cloud-amount values over Spain (point b), have been compared with okta values measured by surface observers, provided by the National Meteorological Service of Spain and converted to percentage of cloud cover. The comparison shows discrepancies lower than $7 \%$ in average.

Global maps of cloud amount were obtained for the analyzed window. The total cloud amount is the sum of mean low-, middle-, and high-level cloud amount. Cloud level was determined from the difference between the equivalent black-body temperature of the surface and the temperature of the cloud top (Minnis and Harrison, 1984). For each pixel, surface temperature was computed as the monthly mean brightness temperature seen by the 

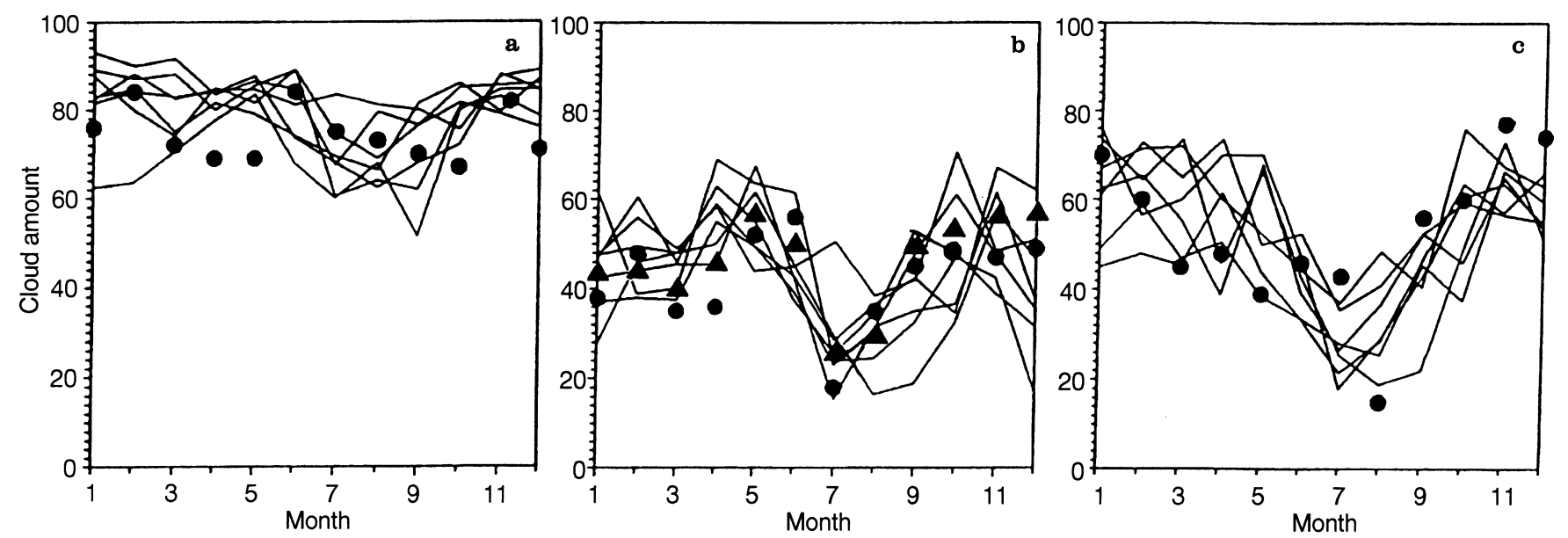

Fig. 4a-c. Monthly evolution of the total cloud amount for points a, b, and c. Solid lines: ISCCP data. Circles: data computed in the present work. Triangles: data measured by surface observers

satellite at the pixel location in clear sky days. A cloud was considered to be low if its top temperature differed by less than $13 \mathrm{~K}$ from the surface temperature. High clouds had differences greater than 39 K. Mid-level clouds gave temperature differences between 13 and $39 \mathrm{~K}$. The temperature thresholds used correspond roughly to altitude ranges of $0-2 \mathrm{~km}, 2-6 \mathrm{~km}$, and greater than $6 \mathrm{~km}$ for the three cloud levels. Low-, middle-, and highlevel cloud amount were computed as the percentage of occurrence of such cloud classes during the month.

In order to simplify the presentations, the months of the year have been grouped into winter (DecemberFebruary), spring (March-May), summer (JuneAugust) and autumn (September-November). The global maps of cloud amount (total, low, mid-level and highlevel) are presented in Figs. 5-8. Each figure includes the cloud data for the four seasons. Also, the annual mean and the root-mean-square (RMS) of the cloud data for the four seasons are presented for each cloud type to provide the mean tendency and the variability of the temporal evolution of the data. Taking into account the high temporal variability from year to year of cloudamount data, the results obtained appear to agree reasonably well with previously published cloud climatology (Hughes, 1984; Warren et al., 1986, 1988; Drake, 1993), although the present data are for only 1 year.

Total cloud amount (see Fig. 5) shows relatively homogeneous values over large spatial scales. Geographical variations are much less smooth over land than over the ocean. This can be due to the fact that surface conditions on land exhibit a much great local variability than over sea. Areas of total cloud amount greater than $60 \%$ are found over almost all the Atlantic ocean and over Europe, for latitudes greater than $42^{\circ}$. The greatest cloud amount $(>75 \%)$ corresponds to the North Atlantic. These high values of cloud cover were maintained throughout the year, as was previously seen in the temporal evolution of Fig. 4. The Saharan area has minimum cloud amount, typically less than $15 \%$, throughout the year. The center and the south of the Iberian peninsula, North Africa, and the Mediterranean
Sea show a greater variability in cloud cover. In these areas the cloud-amount oscillation amplitudes can be greater than $30 \%$, with maximum values in the cold season. The time variability of the total cloud cover could be seen better in the RMS representation. It was found that the most intense changes occurred in the above mentioned areas, especially over the Mediterranean Sea.

From the plots of fractions of the different cloud types, more can be said about the cloud distribution. Cloudiness in sea-surface areas was mainly associated with low-level clouds (stratus, stratocumulus, fog, and cumulus). So, for example, the annual mean of low-level cloud amount shows that the Atlantic Ocean has lowlevel cloud amount greater than $40 \%$. In contrast, land areas have the greatest proportion of high-level clouds, which were practically nonexistent over sea surfaces. The only exception was the Mediterranean Sea, which presents a high probability of occurrence of high-level clouds during autumn. It may be due to the high convective activity in this area during this season. Typical high-level cloud amount over high-latitude land areas ranges from about 8 to $16 \%$ (minimum during summertime and maximum in autumn). Sea surfaces and dry-land areas have covers of around $0-8 \%$. It can also be observed that high-level cloud cover has a less homogeneous spatial distribution than total or low-level cloud cover. It is expected that both satellite and surface observers would have problems estimating cloud amount. Obscuration of low clouds by higher clouds is the most obvious problem for the satellite measures (Rozendaal et al., 1995). The simultaneous presence of both cloud types (Goodman et al., 1990) produces an underestimation of low-level cloud cover seen from satellite as well as a reduction of the high-level cloud amount seen from surface observers.

There are several potential error sources in the process undertaken. Errors in the cloud-amount computations are due to inaccuracies in the cloud-detection methods and errors in the classification of the clouds. The accuracy of the method is not determined by the VIS and/or IR threshold levels as in cloud-detection 

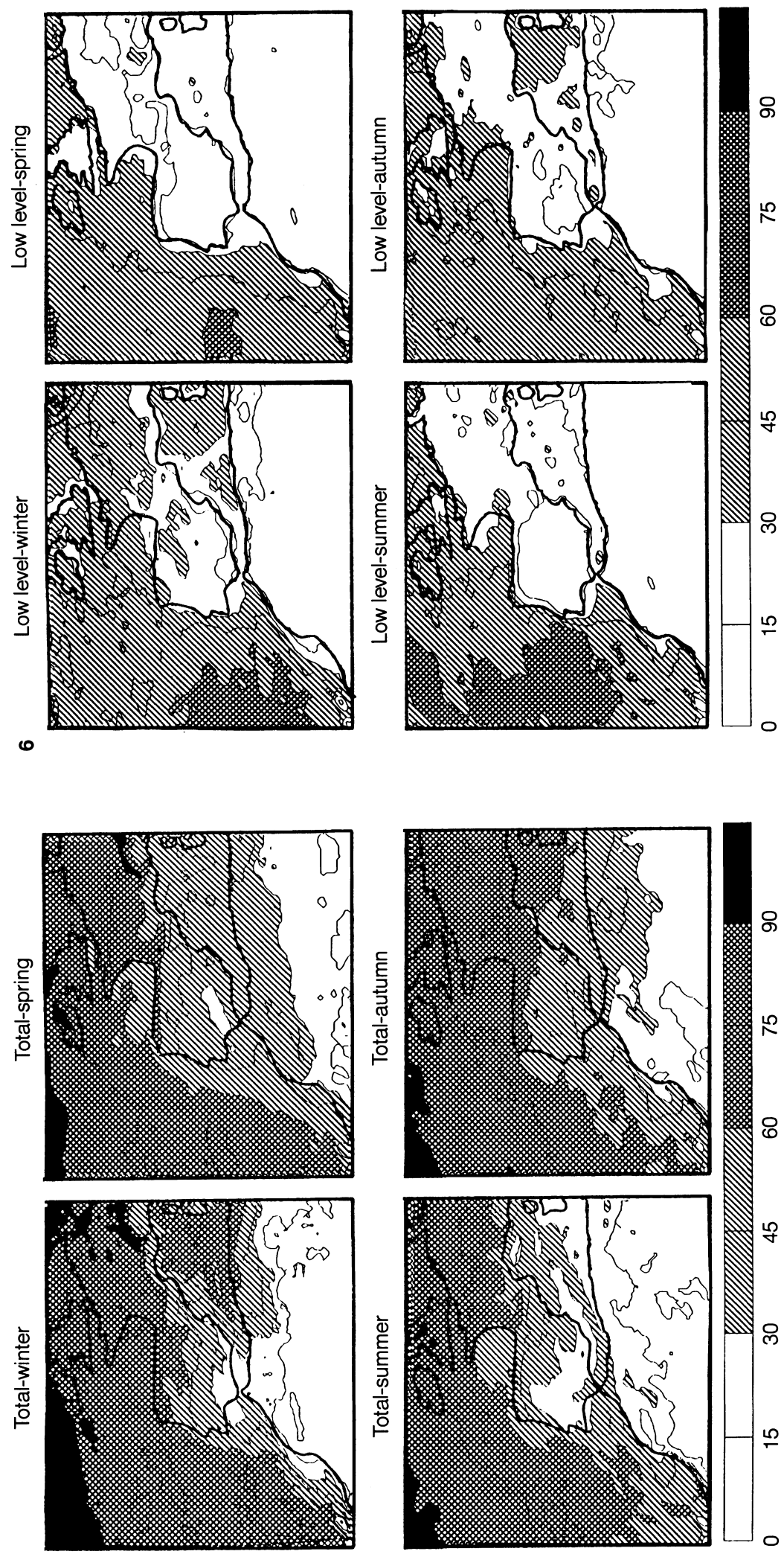
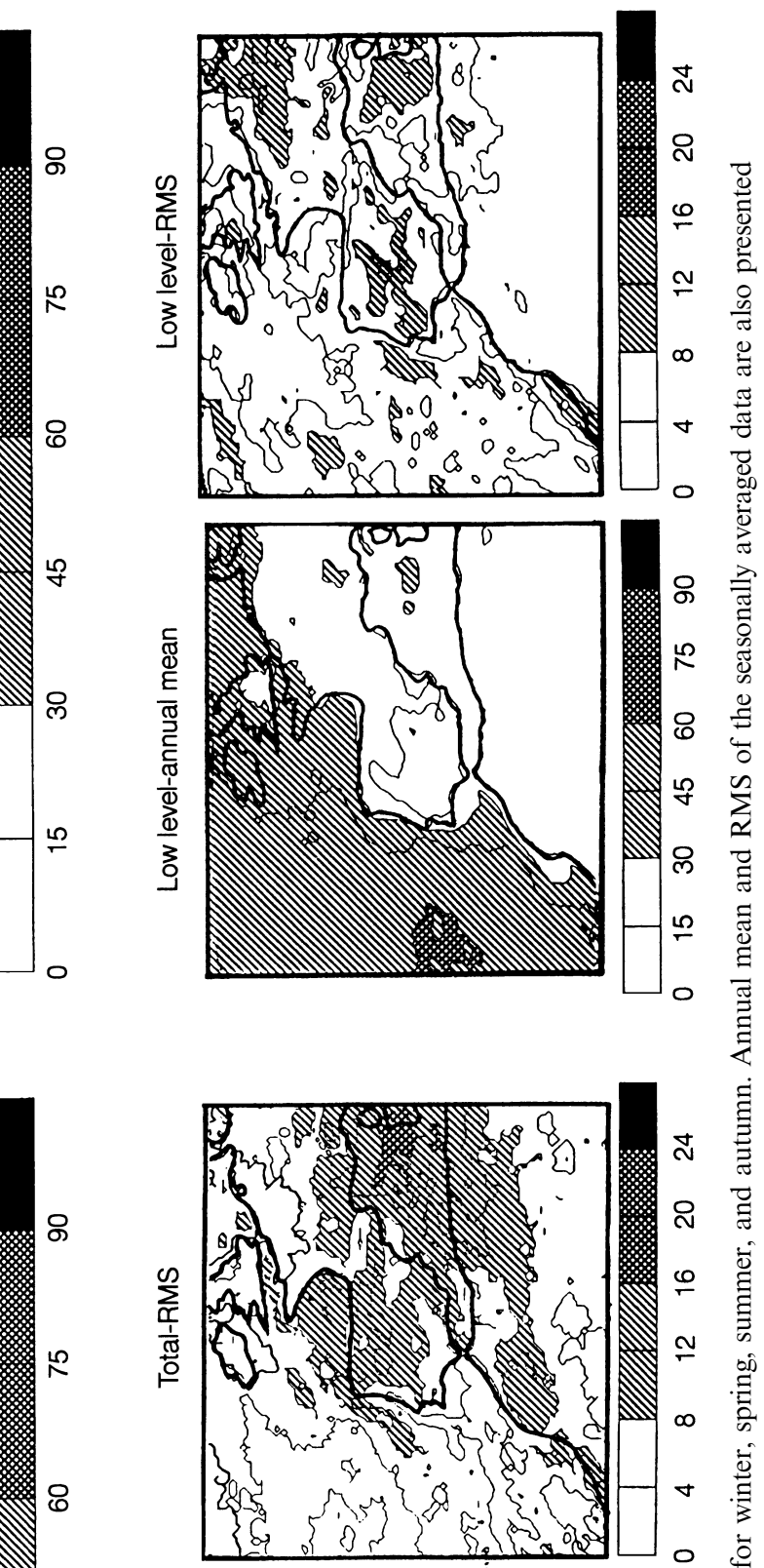

言

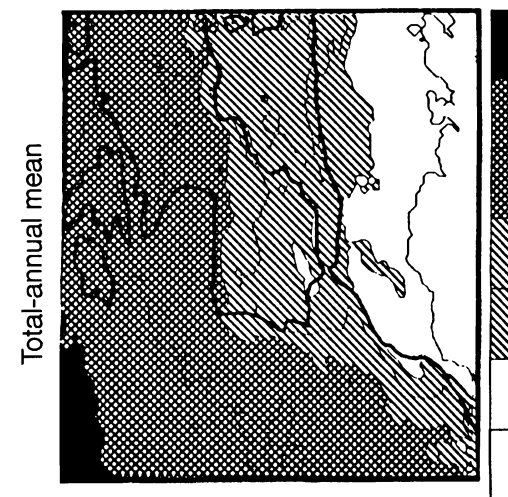

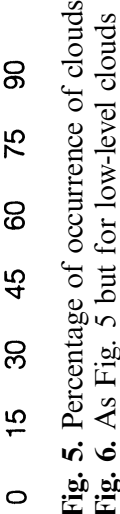



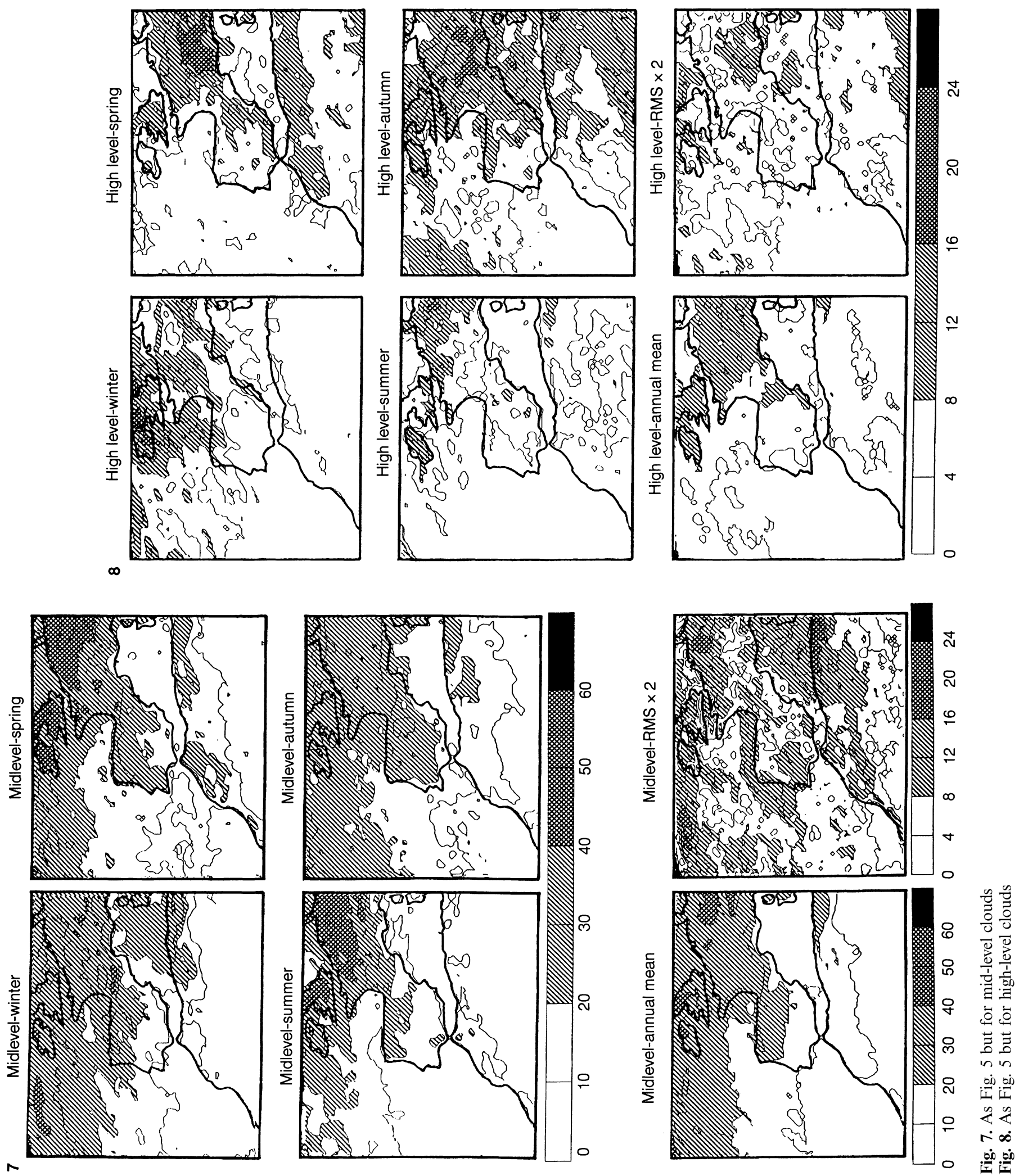
algorithms based on threshold techniques. Cloud-detection errors are mainly produced in scenes where clouds cause few variations of the radiance. In other situations, even where cloudiness is usually persistent, the combined time-variation test and H2D histogram analysis successfully separate the radiance distribution into clear and cloudy categories. It should be noted that the algorithm performs better over oceans than over land. In particular, complex situations over land, like snow cover during winter, are not correctly resolved by the method. Even if a pixel is correctly classified as cloudy, an additional error can occur in its classification as low, mid-level or high-level cloud. The cloud-height calculations are affected both by errors in the determinations of the surface temperature and the cloud-top temperature, mainly due to inaccuracies in the knowledge of the emissivity of the surface. Also, the uncertainties in the vertical temperature gradient produce errors in the height assignment.

Although the cloud analysis is realized mainly at noon, a sensibility test of the cloud detection algorithm was realized for analyzing the effect of the illumination conditions on its accuracy. Concretely, the image data corresponding to May 1995 were fully exploited analyzing the daily variation of the total and low cloudiness. For nighttime images the cloud-detection algorithm must be executed using only IR images. For further validation at cloud detection using both VIS/IR images and using IR only, the cloud amount (total and low) from the IR channel was compared with that derived from the combined VIS and IR images for the hours at which both measurements were available. The analysis, summarized in Fig. 9 for points a, b, and c, indicates a similar behavior of the cloudiness hourly variations determined by VIS/IR images (circles) and IR-only images (solid line). Data obtained by surface observers for point $b$ at the times available are presented as triangles. Hourly evolution of total and low-level cloudiness depict similar trends in the three analyzed points. It is seen that the IR algorithm tends to give lower estimates of cloud amount where low clouds are present. In these zones (mainly over the ocean, point a), cloud-top temperatures are very close to surface temperatures, and the IR channel alone underestimates the cloudiness. The mean difference between VIS/IR and IR-only results is less than $8 \%$.

The mean monthly cloud amount from all hours and the RMS of the data (both for total and low clouds) are presented in Fig. 10. These data are computed using only IR images in order to maintain the homogeneity of the data for day and nighttime. Over the Atlantic Ocean mean cloudiness computed from all hours for May shows lower values than the mean cloudiness from 12 GMT (see Figs. 3 and 4, spring) both for total and low cloudiness. This tendency reverses over Spain. North Africa and the Mediterranean Sea present similar values for total cloudiness and more low-level cloudiness at 12 GMT. Over the other zones both groups of data behave similarly. The diurnal variability of the cloudiness, measured by the RMS of the hourly cloud data, indicates that the strongest variability appears over land for both total and low clouds. Over the ocean the region with largest diurnal cycle is located near the Canary Islands. This greater variability (both for low-level and total cloudiness) also appears in the data of Rozendaal et al. (1995), which analyzed the amplitude of the first harmonic of the diurnal cycle of low cloudiness using ISCCP $2.5^{\circ} \times 2.5^{\circ}$ data.

\section{Conclusions}

In this paper the seasonal distribution of cloud amount was analyzed for a 1-year period using the cloudy pixels determined with a cloud-detection algorithm. The timevariation test is found more adequate than the method based on space variances. Nevertheless, space variance has been used in the detection of broken clouds or cloudy pixels located on the edges of large cloud systems. In general it was observed that cloud properties are systematically different between land and ocean. Oceans have larger cloud amount with lower cloud-top
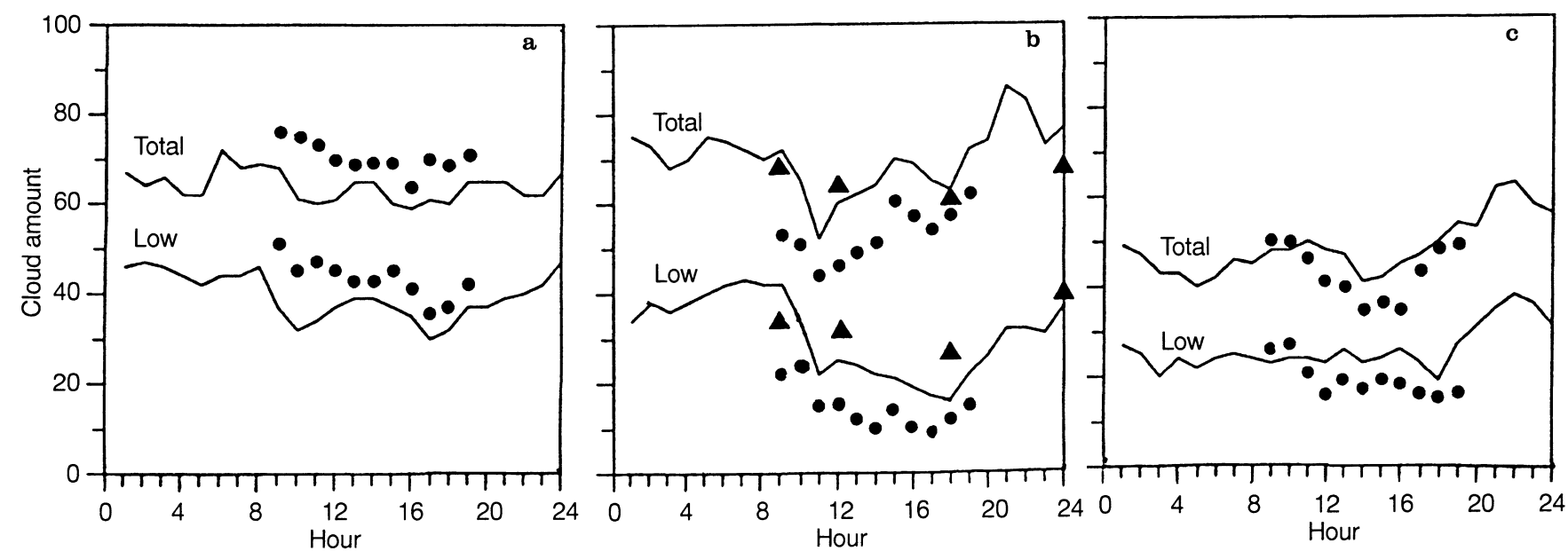

Fig. 9a-c. Diurnal cycle of total and low cloud fraction for May 1995 for points a, b, and c. Solid lines: data from IR images. Circles: data from VIS/IR images. Triangles: data measured by surface observers 

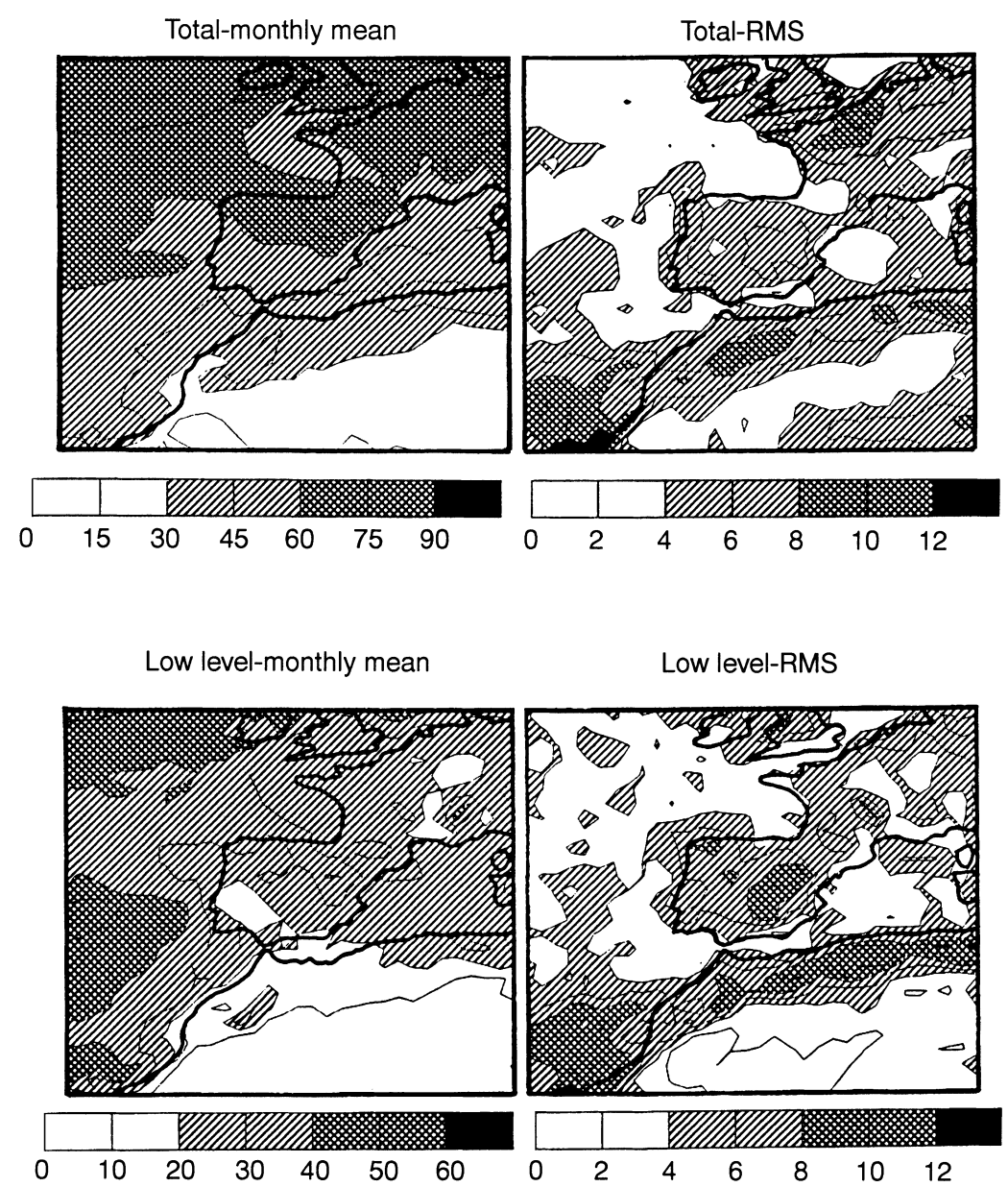

Fig. 10. Mean monthly cloud amount (total and low level) for May 1995 and RMS of the hourly data

altitudes. Some of the largest seasonal variations of cloudiness in the analyzed window occur over the Iberian Peninsula, North Africa, and Mediterranean Sea. The results obtained agree reasonably with other previously published cloud climatology studies.

Acknowledgements. The authors would like to thank to CIRIT (Generalitat de Catalunya) and CICYT (Project AMB94-0948) for the support given to this work. Cloudiness data provided by the Instituto Nacional de Meteorologia (Spain) are also acknowledged.

The Editor-in-chief thanks M. Desbois and another referee for their help in evaluating this paper.

\section{References}

Carlston, B. E., and A. B. Wolf, Spatial and temporal characterization of diurnal cloud variability. Fourth Symp. on Global Change, Anaheim, CA, Am. Meteor. Soc., pp. 98-103, 1993.

Coakley, J. A., and D. G. Baldwin, Towards the objective analysis of clouds from satellite imagery, J. Clim. Appl. Meteorol., 23, 1065-1099, 1984.

Coakley, J. A., and F. P. Bretherton, Cloud cover from high resolution scanner data: detecting and allowing for partially filled fields of view, J. Geophys. Res., 87, 4917-4932, 1982.

Desbois, M., and G. Sèze, Use of space and time sampling to produce representative satellite cloud classifications, Ann. Geophysica, 2, 595-606, 1984.

Drake, F., Global cloud cover and cloud water path from ISCCP C2 data, Int. J. Climatol., 13, 581-605, 1993.
Duvel, J., and L. S. Kaudel, Regional-scale diurnal variations of outgoing infrared radiation observed by Meteosat, J. Clim. Appl. Meteorol., 24, 335-349, 1985.

Goodman, A. H., A. Henderson-Sellers, and K. McGuffie, Climatological contingency probabilities of clouds, Int. J. Climatol., 10, 565-589, 1990.

Henderson-Sellers, A., G. Sèze, F. Drake, and M. Desbois, Surfaceobserved and satellite-retrieved cloudiness compared for the 1983 ISCCP Special Study Area in Europa, J. Geophys. Res., 92, 4019-4033, 1987.

Hughes, N. A., Global cloud climatologies: a historical review, J. Clim. Appl. Meteorol., 23, 724-751, 1984.

Klein, S. A., and D. L. Hartmann, The seasonal cycle of low stratiform clouds, J. Clim., 6, 1587-1606, 1993.

Kondragunta, C. R., and A. Gruber, Diurnal variation of the ISCCP cloudiness, Geophys. Res. Lett., 21, 2015-2018, 1994.

Massons, J., D. Domingo, and J. Grau, Automatic classification of VIS-IR Meteosat images, Comp. Geosci., 22, 1137-1146, 1996.

Minnis, P., and E. F. Harrison, Diurnal variability of regional cloud and clear-sky readiative parameters derived from GOES data. Part II: November 1978 cloud distributions, J. Clim. Appl. Meteorol., 23, 1012-1031, 1984.

Norris, J. R., and C. B. Leovy, Interannual variability in stratiform cloudiness and sea surface temperature, J. Clim., 7, 1915-1925, 1994.

Pankiewicz, G. S., Pattern recognition techniques for the identification of cloud and cloud systems, Meteorol. Appl., 2, 257-271, 1995.

Randall, D. A., J. A. Coackley, C. W. Fairall, R. A. Kropfli, and D. H. Lenschow, Outlook for research on subtropical marine stratiform clouds, Bull. Am. Meteorol. Soc., 65, 1290-1301, 1984. 
Rossow, W. B., and L. C. Garder, Cloud detection using satellite measurements of infrared and visible radiances for ISCCP, J. Clim., 5, 2341-2369, 1993.

Rossow, W. B., and A. A. Lacis, Global, seasonal cloud variations from satellite radiance measurements. Part II: properties and radiative effects, J. Clim., 3, 1204-1253, 1990.

Rossow, W. B., and R. A. Schiffer, ISCCP cloud data products, Bull. Am. Meteorol. Soc., 72, 2-20, 1991.

Rossow, W. B., F. Mosher, E. Kinsella, A. Arking, M. Desbois, E. Harrison, P. Minnis, E. Ruprecht, G. Sèzé, C. Simmer, and E. Smith, ISCCP cloud algorithm intercomparison, J. Clim. Appl. Meteorol., 24, 877-903, 1985.

Rossow, W. B., A. W. Walker, and L. C. Garder, Comparison of ISCCP and other cloud amounts, J. Clim., 6, 2394-2418, 1993.

Rozendaal, M. A., C. B. Leovy, and S. A. Klein, An observational study of diurnal variations of marine stratiform clouds, J. Clim., 8, 1795-1809, 1995.

Saunders, R. W., An automated scheme for removal of cloud contamination from AVHRR radiances over western Europe, Int. J. Rem. Sensing, 7, 867-886, 1986.

Sèze, G., and M. Desbois, Cloud cover analysis from satellite imagery using spatial and temporal characteristics of the data, J. Appl. Meteorol., 26, 287-303, 1987.
Sèze, G., and W. B. Rossow, Time cumulated visible and infrared radiance histograms used as descriptors of surface and cloud variations, Int. J. Rem. Sensing, 12, 877-920, 1991.

(Sèze et al. 1983)

Slingo, J. M., The development and verification of a cloud prediction scheme for the ECMWF model, Q. J. R. Meteorol. Soc., 113, 899-927, 1987.

Thiao, W., and O. M. Turpeinen, Large-scale diurnal variations of tropical cold cloudiness based on a simple cloud indexing method, J. Clim., 5, 173-180, 1992.

Warren, S. G., C. J. Hann, and J. London, Simultaneous occurrence of different cloud types, J. Clim. Appl. Meteorol., 24, 658-667, 1985.

Warren, S. G., C. J. Hann, J. London, R. M. Chervin, and R. L. Jenne, Global distribution of total cloud cover and cloud type amounts over land, NCAR/TN-273 + STR, 1986.

Warren, S. G., C. J. Hann, J. London, R. M. Chervin, and R. L. Jenne, Global distribution of total cloud cover and cloud type amounts over the ocean, NCAR/TN-317+STR, 1988. 